\title{
Grundprobleme und Perspektiven des Gentechnikrechts
}

\author{
Michael Kloepfer und Stella Doerenbach
}

(c) Der/die Autor(en) 2021. Dieser Artikel ist eine Open-Access-Publikation.

Der vorliegende Beitrag zielt darauf ab, einen Überblick über aktuelle Entwicklungen des Gentechnikrechts zu verschaffen. Neben einigen (immer noch bestehenden) Grundproblematiken im Spannungsfeld zwischen Vorsorgeprinzip und Innovationsbestreben, gibt es sowohl Neuerungen in der europäischen Rechtsprechung, als auch in der technischen und gesellschaftlichen Entwicklung, die vorgestellt und in Kontext gesetzt werden. Einer engeren Untersuchung werden dabei u. a. das Mutagenese-Urteil des EuGH aus dem Jahr 2018 und sogenannte „Gene-Drives“ unterzogen.

\section{Einführung}

Am 29. April 2021 hat die EU-Kommission eine im Auftrag des EU-Rates vorgenommene Studie veröffentlicht, die konstatiert, dass die Regulierung von Gentechnik in Europa überarbeitet werden soll. ${ }^{1}$ Betont wird, dass der derzeit geltende Rechtsrahmen für genveränderte Organismen aus dem Jahr 2001 nicht mehr für die heutige innovative Technologie geeignet sei und nun ein breit angelegter und offener Konsultationsprozess eingeleitet werden soll, um für neue Verfahren neue Regeln einführen zu können. ${ }^{2}$ Auch in Hinblick auf den Welternährungsgipfel der Vereinten Nationen, der im September 2021 in New York stattfand, müssen die rechtlichen Rahmenbedingungen in Bezug auf gentechnische Fragen überdacht und unter Umständen überarbeitet werden, um sich diesbezüglich klar auf internationaler Ebene positionieren zu können. ${ }^{3}$

Anders als beim Atomrecht, bei dem 2011 der Ausstieg aus der Atomenergie festgeschrieben wurde, scheiterte der deutsche Gesetzgeber bei dem Versuch, den Anbau europaweit bereits zugelassener gentechnisch veränderter Pflanzen national wieder zu verbieten. ${ }^{4}$ Das Gentechnikrecht stellt sich somit in Abgrenzung zu anderen Risikotechnologien, wie dem Atomrecht, als umweltrechtliches Teilrechtsgebiet dar, welches sich gerade im Hinblick auf fortschreitende technische Möglichkeiten auch in Zukunft weiterentwickeln wird und rechtliche Anpassungen wahrscheinlich unumgänglich macht. Im Folgenden soll zunächst in die immer noch bestehenden rechtlichen Grundprobleme eingeführt werden, um sodann die relevantesten Neuerungen der letzten Jahre in Bezug auf das Gentechnikrecht und die damit einhergehenden Probleme und Perspektiven betrachten zu können. Nicht behandelt wird in diesem Aufsatz das besonders umstrittene Gebiet der Humangenetik, das ganz eigene, nämlich menschenbezogene, Maßstäbe hat.

\section{Begriff und Wesen der Gentechnik}

Die Gentechnik ist ein Teilgebiet der Biotechnologie. ${ }^{5}$ Biotechnologie ist jede technologische Anwendung, die

\section{Prof. Dr. Michael Kloepfer,}

Emeritierter Prof. des Offentlichen Rechts

an der Juristischen Fakultät,

(u.a.) Leiter des Forschungszentrums Umweltrecht (FZU),

Humboldt-Universität zu Berlin,

Berlin, Deutschland

Stella Doerenbach, Wissenschaftliche Mitarbeiterin am FZU, Doktorandin am Lehrstuhl für Rechtsphilosophie

und Öffentliches Recht,

Christian-Albrechts-Universität zu Kiel,

Kiel, Deutschland biologische Systeme, lebende Organismen oder Produkte daraus benutzt, um Erzeugnisse oder Verfahren für eine bestimmte Nutzung herzustellen oder zu verändern. ${ }^{6}$ Dabei stellt die Gentechnik die Summe aller Methoden zur Isolierung, Charakterisierung und gezielter Veränderung und Übertragung von Erbgut dar. ${ }^{7}$

Unterschieden werden kann zudem zwischen dem Verfahren der klassischen Gentechnik, bei der es dem Zufall überlassen wird, wie und an welcher Stelle sich das gewünschte Gen in das Genom einfügt und dem Verfahren des sog. Genome Editing, worunter auch die Gen-Schere CRISPR $/ \mathrm{Cas}^{8}$ fällt, bei dem ziemlich sicher festgelegt werden kann, wo die beabsichtigte Änderung platziert werden soll. ${ }^{9}$

Die Gen- und Biotechnik sind Schlüsseltechnologien des 21. Jahrhunderts. ${ }^{10}$ Der Anwendungsbereich ist weit. Nicht nur das Verändern von Erbgut zur Nutzpflanzengewinnung, wie Getreide, steht im Raum, sondern auch das Klonen von Tieren zur Lebensmittelversorgung oder das Etablieren von sog. Gene-Drives ${ }^{11}$, einem Mittel der synthetischen Energie, mit deren Hilfe verhindert werden könnte, dass Mücken Malaria übertragen ${ }^{12}$ und alle damit einhergehenden ethischen und rechtlichen Fragen werden vehement diskutiert. Der Anwendungsbereich erstreckt sich auch in den privaten Bereich, beispielsweise durch so-

1) Komm., Pressemitt. v. 29.4.2021, Studie der EU-Kommission zu Gentechnik: Für neue Verfahren braucht es neue Regeln, IP/21/1985.

2) Komm., Pressemitt. v. 29.4.2021, Studie der EU-Kommission zu Gentechnik: Für neue Verfahren braucht es neue Regeln, IP/21/1985.

3) Bundesministerium für Ernährung und Landwirtschaft: Ergebnisbericht der Tagung des Rates (Landwirtschaft und Fischerei) am 26.-27.5.2021 in Brüssel, Stand 28.10.2021, abrufbar unter https://www.bmel.de/DE/themen/landwirtschaft/eu-agrarpolitik-und-foerderung/gap/agrarrat-05-2021.html.

4) Hansmann/König, in: Landmann/Rohmer, Umweltrecht, Stand 1. 5. 2021, 12. BImSchV vor $\$ 19$, Rdnr. 13.

5) Kloepfer/Durner, Umweltschutzrecht, 3. Aufl. 2020, \$17, Rdnr. 1.

6) BT-Drs.10/6775, S. 7.

7) Schell/Mohr, Biotechnologie - Gentechnik, 1995, S. 1.

8) Mit dieser Methode können Gene gezielt eingefügt, entfernt oder ausgeschaltet werden, siehe weiter unter: Transparenz Gentechnik: CRISPR/Cas-System, Stand 28.10.2021, abrufbar unter https://www.transgen.de/lexikon/1845.crispr-cas.html\#: : text $=$ Neue $\% 2 \mathrm{C} \% 20$ molekularbiologische $\% 20$ Methode $\% 2 \mathrm{C} \%$ 20 um\%20DNA,funktioniert $\% 20$ grunds\%C3\%A4tzlich\%20bei\% 20allen\%20Organismen.

9) Bundesministerium für Umwelt, Naturschutz und nukleare Sicherheit: Was ist der Unterschied zwischen Genome Editing und klassischer Gentechnik?, Stand 28.10.2021, abrufbar unter https://www.bmu.de/service/haeufige-fragen-faq/faq-zu-gentechnik/.

10) Kloepfer, Umweltrecht, 4. Aufl. 2016, $\$ 20$, Rdnr. 1.

11) Bei dieser Methode werden bestimmte Gene in natürliche Populationen eingebracht und eigenständig auf alle Individuen ausgebreitet, Akademie der Naturwissenschaften Schweiz (SCNAT): Gene Drive, Stand 28.10.2021, abrufbar unter https://naturwissenschaften.ch/synthetic-biology-explained/applications/gene drive.

12) Akademie der Naturwissenschaften Schweiz (SCNAT): Gene Drive, Stand 28.10.2021, abrufbar unter https://naturwissenschaften.ch/synthetic-biology-explained/applications/gene_ drive. 
genannte „Do-It-Yourself-Kits“, mit welchen Privatpersonen mit Gen-Scheren experimentieren können.

\section{Gentechnik als Risikotechnologie: Grundproblematiken}

\subsection{Spannungsverhältnis zwischen dem Vorsorgeprinzip und Innovationsermöglichung}

Das rechtliche Grundprinzip, welches vor allem beim Gentechnikrecht von großer Bedeutung ist, ist das Finden eines Ausgleichs zwischen dem Vorsorgeprinzip (Art. 191 Abs. 2 AEUV) auf der einen und Innovationsbestrebungen auf der anderen Seite. Gentechnische Neuerungen sollen auf der einen Seite gefördert werden, auf der anderen Seite bedarf es eines hohen Schutzniveaus der Bevölkerung. ${ }^{13}$ Dieses bestehende Spannungsverhältnis, nämlich den Schutz vor Risiken einerseits und die Förderung von Innovationen andererseits zu regeln, ist eine grundlegende Problematik des Gentechnikrechts.

Beispielsweise könnte gentechnisch verändertes Saatgut Trockenheit besser standhalten und widerstandsfähigere und schädlingsresistentere Pflanzen gegen steigende Lebensmittelpreise und Hungersnöte signifikante, positive Veränderungen vornehmen. ${ }^{14}$

Zudem bedarf es einer starken Regulierung, um Risiken effektiv $\mathrm{zu}$ verhindern und dem unionsrechtlichen Grundprinzip der Vorsorge und Vorbeugung Rechnung zu tragen. ${ }^{15}$ Gerade bei der Weiterentwicklung und Anwendung neuer gentechnischer Praktiken ist Vorsicht geboten, da die Besonderheit bei der Gentechnik gerade darin liegt, dass es nach wie vor Unsicherheiten über die Langzeitwirkungen und -risiken der Gentechnik bestehen. ${ }^{16}$ Verhindert werden soll, dass beispielsweise gentechnisch veränderte Organismen in die Umwelt gelangen, welche dort unkontrollierbare Prozesse in Gang setzen und die Umwelt nachhaltig verändern können. ${ }^{17}$ Auch im Hinblick darauf, dass sich die technischen Entwicklungen innerhalb der Gentechnik stetig weiterentwickeln, bedarf es kontinuierlicher Beobachtung der technischen Entwicklungen und gegebenenfalls rechtlicher Anpassungen. $\mathrm{Zu}$ beachten ist zudem, dass in Bezug auf die Risikovorsorge keine bestimmte Schwelle erreicht werden muss. Das Vorsorgeprinzip greift bereits ein, wenn es die begründete Möglichkeit, bzw. eine abstrakte Besorgnis besteht, dass Umweltbeeinträchtigungen und Grundrechtsgefahren eintreten könnten. ${ }^{18}$ Dies ist vor allem in Bezug auf das Gentechnikrecht von Bedeutung, da aufgrund des rasant steigenden technologischen Fortschritts oft nicht mit Sicherheit gesagt werden kann, ob und gegebenenfalls wo die Risiken der neuartigen Entwicklungen liegen, auch weil manchmal aufgrund der rasanten Entwicklung keine vorherigen Langzeitstudien durchgeführt werden können.

\subsection{Biosecurityfragen: sog. Dual-Use-Dilemma oder Dilemma des doppelten Verwendungszwecks}

Vor diesem Hintergrund muss auch der Dual-Use, also der doppelte Verwendungszweck beachtet werden. Dieser Begriff wurde ursprünglich zur Unterscheidung zwischen militärischer und ziviler Anwendungen benutzt. ${ }^{19}$ Der Begriff des doppelten Verwendungszwecks findet aber auch mehr und mehr Anwendung bei biochemischen Prozessen in Zusammenhang mit friedlicher Verwendung von biochemischen Systemen, die dem Menschen dienen, aber auch in potentiell missbräuchlicher Absicht eingesetzt werden können. ${ }^{20}$ Beispielsweise wurde von der USArmee - vielfach kritisiert - das spanische Grippevirus, welches 27 Millionen Menschenleben kostete, nachgezüchtet. ${ }^{21}$ Vor dem Hintergrund des doppelten Verwendungszwecks stellt sich die Frage, ob bestimmte gentech- nische Informationen in die Öffentlichkeit geraten dürfen oder ob das Fehlgebrauchs- und Missbrauchspotential zu hoch ist.

\subsection{Probleme durch grenzübergreifende Wirkung der Gentechnik}

Hinzu tritt das Problem der internationalen Dimension des Gentechnikrechts; andere Staaten müssen notwendigerweise mit den veränderten umweltbedingten Folgen leben, da die Umwelt nicht an den Ländergrenzen Halt macht. Manche gentechnisch veränderten Manipulationen lassen sich nicht mehr rückgängig machen. Sollte beispielsweise ein Land die sog. Genes Drives zulassen, welche gentechnische Kettenreaktionen in Bezug auf das Erbgut auszulösen vermögen, können diese durch Pollen oder Mücken etc. über Staatsgrenzen hinweggetragen werden. ${ }^{22}$ Diese speziell (aber nicht nur) bei der Gentechnik auftretende Gefahr ist eines der Grundprobleme dieses Gebiets und ihr gilt besonderer Beachtung vor allem in Bezug auf internationale, völkerrechtliche und europarechtliche Regelungsmechanismen. ${ }^{23}$

\subsection{Problematik der „richtigen“ Regulierungsphilosophie: produktbezogen oder verfahrensbezogen?}

Altbekannt scheint der Streit um die richtige Regulierungsphilosophie in Bezug auf das Gentechnikrecht. ${ }^{24}$ Zwei Möglichkeiten können unterschieden werden: Zum einen der produktbezogene Regulierungsansatz, zum anderen der verfahrensbezogene. Beim produktbezogenen Regulierungsansatz unterliegt lediglich das am Ende hergestellte Produkt der rechtlichen Bewertung, wohingegen beim verfahrensbezogenen Ansatz die Organismen aufgrund des Herstellungsprozesses beurteilt werden. Welchen der beiden Ansätze man verfolgt, hat Auswirkungen auf die letztliche rechtliche Wertung des gentechnisch modifizierten Gesamtproduktes, sowie praktische Auswirkungen auf Forschung und Industrie..$^{25}$ Beim produktbezogenen Regulierungsansatz stellt sich das relevante Fol-

13) Seitz, EuZW 2018, 757.

14) Bühl, Auf dem Weg zur biomächtigen Gesellschaft? Chancen und Risiken der Gentechnik, 2009, 23.

15) Seitz, EuZW 2018, 757.

16) Kloepfer, Umweltrecht, 4. Aufl. 2016, 1764.

17) Kloepfer/Durner, Umweltschutzrecht, 3. Aufl. 2020, \$17, Rdnr. 2.

18) Kahl, in: Streinz, EUV/AEUV, 3. Aufl. 2018, Art. 191, Rdnr. 78 , nicht ausreichend für Schutzmaßnahmen ist allerdings, wenn sich diese auf rein hypothetische Annahmen stützen.

19) Schweizerische Akademie der Technischen Wissenschaften (SATW): Synthetische Biologie. Eine neue Ingenieurwissenschaft entsteht, Stand 28.10.2021, abrufbar unter https://www. satw.ch/fileadmin/user_upload/documents/02 Themen/06 Rohstoffe/SATW-Synthetische_Biologie-DE.pdf.

20) Schweizerische Akademie der Technischen Wissenschaften (SATW): Synthetische Biologie. Eine neue Ingenieurwissenschaft entsteht, Stand 28.10.2021, abrufbar unter https://www. satw.ch/fileadmin/user_upload/documents/02_Themen/06_ Rohstoffe/SATW-Synthetische_Biologie-DE.pdf.

21) Deutschlandfunk: US-Armee züchtet die "Spanische Grippe“ nach, Stand 28.10.2021, abrufbar unter https://www.deutschlandfunk.de/meldungen-liste-forschung-aktuell.1508.de.html? drn:news_id $=77191$.

22) Umweltinstitut München e.V.: Gene Drive: Gentechnik jetzt auch an wildlebenden Arten?, Stand 28.10.2021, abrufbar unter http://www.umweltinstitut.org/themen/gentechnik/genedrive.html.

23) Eingehend dazu: Vöneky, International Standard Setting in Biomedicine - Foundations and New Challenges, in: German Yearbook of international law 61, 2018.

24) Spranger, NJW 2018, 2929.

25) Andersen/Schreiber, in: Vöneky, Freiburger Informationspapiere zum Völkerrecht und Öffentlichen Recht, Ausgabe 11/2019: „Genome Editing“" vor dem EuGH und seine Folgen, S. 10. 
geproblem der nachträglich fehlenden Nachweisbarkeit des Verfahrens. Beim Verfahren der gerichteten Punktmutagenese beispielsweise, eine neue Züchtungsmethode, kann durch nachträgliche Anwendung klassischer Mutationsverfahren das Eingreifen des Menschen unnachweisbar überdeckt werden. ${ }^{26}$ Der Züchter müsste eigeninitiativ deklarieren, welche Verfahren er genau angewendet hat. Tut er dies nicht und würde das Lebensmittel lediglich als ein mit klassischen Mutageneseverfahren erzeugtes Produkt klassifiziert, dann greift die Anwendung der Freisetzungsrichtlinie gem. Art. 3 RL 2001/18/EG, welche die Anwendung des Gentechnikrechts nach \$3 Nr. 3b Gentechnikgesetz (GenTG) entfallen ließe. ${ }^{27}$ Aufgrund der mangelnden Möglichkeit der Nachweisbarkeit besteht somit beim produktbezogenen Verfahren die Gefahr, dass bestehende Regulierungen und Genehmigungserfordernisse des deutschen Gentechnikrechts unterlaufen werden. ${ }^{28}$

Eben dies war in Hinblick auf die Frage, ob der Begriff der „,gentechnisch veränderten Organismen“ (GVO) prozessorientiert oder ergebnisorientiert auszulegen sei, lange umstritten. Der EuGH hat nun aber entschieden, dass es bei der Einstufung als GVO nicht auf das Ergebnis, sondern nur auf das Verfahren ankommt. Deutschland folgt somit seit dem wegweisenden Urteil des EuGH aus dem Jahr $2018^{29}$ implizit dem prozessbasierten Ansatz: Hätte das Ergebnis eines neuen gentechnologischen Verfahrens somit auch mithilfe herkömmlicher Züchtungsmethoden erreicht werden können, ist dies irrelevant und die Organismen unterliegen dennoch GVO-Regulierungen. ${ }^{30}$ Eine Umgehung der Kennzeichnungspflicht ist damit erheblich erschwert worden, da alle Pflanzen - egal ob durch ein konventionelles oder neuartiges Mutagenese-Verfahren verändert - in den Anwendungsbereich der Richtlinie fallen und damit streng reguliert sind. ${ }^{31}$

\subsection{Gesellschaftspolitische Akzeptanz der Gentechnik}

Nicht zu unterschätzen ist die gesellschaftspolitische Diskussion wenn es um genveränderte Lebensmittel geht. In Deutschland lehnen $79 \%$ der Bevölkerung die Fütterung von Nutztieren mit genverändertem Futter ab. ${ }^{32} 75 \%$ sind zudem der Überzeugung, dass der Mensch kein Recht habe, Pflanzen und Tiere gentechnisch zu verändern. ${ }^{33}$ Generell überwiegt die Skepsis der Bevölkerung, wenn es um genveränderte Organismen geht. Laut der letzten Naturbewusstseinsstudie aus dem Jahr 2019 vertrauen nur 36\% der befragten Wissenschaftlerinnen und Wissenschaftler, wenn diese bestätigen, dass die neuen gentechnischen Verfahren sicher sind. ${ }^{34}$ Dies kann durchaus ein Problem darstellen. Wenn der Rückhalt und das Vertrauen der Bevölkerung bezüglich der Gentechnik nicht vorhanden ist, ist es sowohl für den Gesetzgeber als auch für die Judikative schwer, zukunftsweisende und naturwissenschaftlich fundierte Gesetze zu erlassen und Urteile zu fällen, die auf breite Akzeptanz in der Bevölkerung stoßen. Auch könnte die Bevölkerung potentiell dazu in der Lage sein, Forschungen zu verlangsamen oder zu stoppen. ${ }^{35}$ Hier können bildungspolitische Maßnahmen ergriffen werden um wissenschaftliche Zusammenhänge und Resultate ausreichend mit der Bevölkerung zu kommunizieren. ${ }^{36}$

\section{Aktuelle Probleme und Neuerungen im letzten Jahrzehnt}

Die eben dargelegten Grundprobleme äußern sich vor allem in den Zielkonflikten, unter anderem in der Rechtsprechung des EuGH, welche sich im Jahr 2018 mit der Auslegung der sog. Mutagenese beschäftigt hat.

\subsection{Mutagenese-Urteil des EuGH aus dem Jahr 2018}

Das Vorabentscheidungsverfahren, welches am 17.10.2016 vom Conseil d'État an den EuGH gerichtet wurde, ${ }^{37}$ be- handelte die Auslegung des Begriffs der Mutagenese im Sinne der Freisetzungsrichtlinie 2001/18/EG und damit die Anwendbarkeit des Gentechnikrechts auf bestimmte neue Züchtungstechnologien. ${ }^{38}$ Geklagt hatte die Confédération paysanne, ein französischer Landwirtschaftsverband zusammen mit acht weiteren Verbänden, da sie sich besorgt sahen über die sich mit der Zeit veränderten Mutageneseverfahren, welche Gesundheitsgefahren für Mensch und Tier bilden könnten. ${ }^{39}$ In Frankreich war nämlich das Mutagenese-Verfahren CRISPR/Cas9 von den Beschränkungen der GVO-Richtlinie ausgenommen und damit nicht streng reglementiert. Dieses sog. Mutagenese-Urteil ist in Schrifttum und Gesellschaft aufgrund der dort vorgenommen Auslegung des Mutagenesebegriffs hoch umstritten und hat nach Veröffentlichung am 25.7.2018 eine Diskussion ausgelöst. ${ }^{40}$

\subsubsection{Biologischer Hintergrund der Mutagenese}

Die Mutagenese ist eine Züchtungstechnik, bei der durch Strahlen, Chemikalien oder durch ein Enzym, das das Erbgut aufschneidet, die genetische Ausstattung von Pflanzen

26) Faltus, Stellungnahme im Rahmen der Sachverständigenanhörung durch den Ausschuss für Ernährung und Landwirtschaft im Deutschen Bundestag, Stand 28.10.2021, abrufbar unter https:// www.bundestag.de/resource/blob/666020/3ce7f0cd0ebdc3dd 6dc19b3473a77790/Stellungnahme_A_Faltus-data.pdf, S. 5.

27) Faltus, Stellungnahme im Rahmen der Sachverständigenanhörung durch den Ausschuss für Ernährung und Landwirtschaft im Deutschen Bundestag, Stand 28.10.2021, abrufbar unter https:// www.bundestag.de/resource/blob/666020/3ce7f0cd0ebdc3dd 6dc19b3473a77790/Stellungnahme_A_Faltus-data.pdf, S. 5.

28) Faltus, Stellungnahme im Rahmen der Sachverständigenanhörung durch den Ausschuss für Ernährung und Landwirtschaft im Deutschen Bundestag, Stand 28.10.2021, abrufbar unter https:// www.bundestag.de/resource/blob/666020/3ce7f0cd0ebdc3dd 6dc19b3473a77790/Stellungnahme_A_Faltus-data.pdf, S. 5.

29) EuGH, Urt. v. 25.7.2018 - C-528/16, ECLI:EU:C:2018:583.

30) Seitz, EuZW 2018, 758.

31) Andersen/Schreiber, in: Vöneky, Freiburger Informationspapiere zum Völkerrecht und Öffentlichen Recht, Ausgabe 11/2019: „Genome Editing“ vor dem EuGH und seine Folgen, S. 11.

32) Mechel, in: Koch/Hofmann/Reese, Handbuch Umweltrecht, 5. Aufl. 2018, §11, Rdnr. 24.

33) Mechel, in: Koch/Hofmann/Reese, Handbuch Umweltrecht, 5. Aufl. 2018, \$11, Rdnr. 24.

34) Bundesministerium für Umwelt, Naturschutz und nukleare Sicherheit (BMU)/Bundesamt für Naturschutz (BN): Informationen zur Naturbewusstseinsstudie, Stand 28.10.2021, abrufbar unter https://www.bfn.de/fileadmin/BfN/gesellschaft/ Dokumente/infopapier_naturbewusstseinsstudie_2019 bf.pdf, S. 12 .

35) Schweizerische Akademie der Technischen Wissenschaften (SATW): Synthetische Biologie. Eine neue Ingenieurwissenschaft entsteht, Stand 28.10.2021, abrufbar unter https://www. satw.ch/fileadmin/user_upload/documents/02_Themen/06_ Rohstoffe/SATW-Synthetische_Biologie-DE.pdf, S. 14.

36) Schweizerische Akademie der Technischen Wissenschaften (SATW): Synthetische Biologie. Eine neue Ingenieurwissenschaft entsteht, Stand 28.10.2021, abrufbar unter https://www. satw.ch/fileadmin/user_upload/documents/02_Themen/06_ Rohstoffe/SATW-Synthetische_Biologie-DE.pdf, S. 14.

37) Vorabentscheidungsersuchen des Conseil d'Etat (Frankreich), Rechtssache C-528/16 eingereicht am 17.10.2016.

38) Einzelfragen zu den Auswirkungen des Urteils des Europäischen Gerichtshofes v. 25. 7.2018 zur Auslegung des Begriffs der Mutagenese im Sinne der Freisetzungsrichtlinie 2001/18/EG, Ausarbeitung ED 5 - $3000-110 / 18$, S. 5.

39) Komm., Pressemitt. v. 25.6.2018, Durch Mutagenese gewonnene Organismen sind genetisch veränderte Organismen (GVO) und unterliegen grundsätzlich den in der GVO-Richtlinie vorgesehenen Verpflichtungen, 111/18.

40) Tagesschau, Neue Gentechnik nur unter strengen Auflagen, Stand 28.10.2021, abrufbar unter https://www.tagesschau.de/ ausland/eugh-gentechnik-recht-101.html. 
und Tieren verändert wird, wobei anders als bei der Transgenese kein fremdes Erbgut in einen lebenden Organismus transferiert wird. ${ }^{41}$ Als Mutagenese wird daher das artifizielle Auslösen von Mutationen bezeichnet. ${ }^{42}$ Dies kann durch konventionelle und zufällige (sog. ungerichtete Verfahren) oder auch zielgerichtet durch neuartige Mutageneseverfahren (sog. gerichtete Verfahren) entstehen. ${ }^{43}$

\subsubsection{Einordnung der Mutagenese im Gentechnikrecht}

Sind durch Mutagenese erstellte Organismen gentechnisch veränderte Organismen (GVO), dann fallen sie unter die strenge Regulierung des Gentechnikrechts. Allerdings können bestimmte Verfahren davon ausgenommen sein. Die Einordnung der Mutagenese im Gentechnikrecht erfolgt somit durch ein Regel-Ausnahme-Verhältnis: Handelt es sich bei der Mutagenese um GVO, sind die Organismen streng reguliert (= Regel), fallen sie unter den Mutagenese-Ausnahmetatbestand des Art. 3 Abs. 1 i. V.m. Anhang Abs. 1 B Nr. 1 FreisetzungsRL der Richtlinie 2001/18/ $\mathrm{EG}$, dann sind sie nicht streng reguliert (= Ausnahme). Die bisher praktizierten Mutageneseverfahren fielen unter die Ausnahme und waren somit nicht streng reguliert.

\subsubsection{Problemaufriss}

Die Frage war nun, ob die Mutagenese-Ausnahme nur für ungerichtete oder auch für gerichtete Verfahren gilt. ${ }^{44}$ Sollten nämlich auch die neuen, gerichteten Mutageneseverfahren unter die Ausnahme fallen, wären sie ebenso wie die bisher etablierten Mutageneseverfahren nicht streng reguliert. Wie der Begriff der Mutagenese somit auszulegen ist, ist von großer Bedeutung und von weitem gesellschaftlichem und politischem Interesse, was der Grund für die große Beachtung des Mutagenese-Urteils aus dem Jahr 2018 war.

\subsubsection{Entscheidung des EuGH}

Das Ergebnis des Vorabentscheidungsverfahrens des EuGH aus dem Jahr 2018 war nun, dass der Anwendungsausschluss der Richtlinie nur für alle Mutageneseverfahren gilt, die vor Einführung der Richtlinie etabliert waren und somit als sicher galten. ${ }^{45}$ Sogenannte Zufallsmutagenesen beispielsweise, welche dem konventionellen Mutageneseverfahren unterkategorisiert werden, fallen nicht unter den Begriff GVO und sind damit vom Anwendungsbereich der Richtlinie ausgenommen. ${ }^{46}$ Das liegt daran, dass solche „,klassischen Verfahren“ als sicher gelten und damit nicht unter die strengen Regularien des Gentechnikrechts fallen. ${ }^{47}$ Hier ist weder eine gentechnikrechtliche Zulassung, noch eine Kennzeichnung eines solchen Verfahrens notwendig. $^{48}$

Nach Erlass der Richtlinie im Jahr 2001 eingeführte neue Mutageneseverfahren fallen laut EuGH nun aber in den Anwendungsbereich, also in die Regel und werden nicht ausgenommen. ${ }^{49}$ Damit fällt beispielsweise auch der Anbau von Gen-Scheren ${ }^{50}$ mutagenisierter Pflanzen unter die aufwändige Zulassung durch das Gentechnikrecht ${ }^{51}$, sowie Punktmutationen, welche eine höhere Genauigkeit und bessere Steuerbarkeit im Gegensatz zu älteren Mutageneseverfahren aufweisen. ${ }^{52}$

\subsubsection{Naturwissenschaftliche Kritik}

Bis zu diesem Mutagenese-Urteil des EuGH aus dem Jahr 2018 vertrat das Bundesamt für Verbraucherschutz und Lebensmittelsicherheit (BVL) die Ansicht, dass Organismen, die durch gezielte Mutagenese entstanden sind, entweder bereits keine GVO seien und damit überhaupt nicht unter die EU-Freisetzungsrichtlinie fallen könnten oder - sollte man sie doch als GVO werten - die gezielten Mutageneseverfahren unter den Ausnahmetatbestand der Freisetzungsrichtlinie fielen. ${ }^{53}$ Grund für diese Praxis war der wesentliche Unterschied zwischen der Mutagenese und Transgenese und die Tatsache, dass sich Mutationen, die mittels gerich- teter Mutagenese erzeugt wurden, sich auf Ebene der DNA qualitativ nicht von Mutationen unterscheiden, die mittels Zufallsmutagenese erzeugt wurden. ${ }^{54}$

Zudem würden seit Jahrzehnten durch chemische Agenzien oder durch (radioaktive) Strahlung Mutationen in der DNA von agrarischen Nutzpflanzen erzeugt, welche auch nicht unter den Regelungsgegenstand des Gentechnikrechts fielen und die Ergebnisse von Menschen als Nahrungsmittel konsumiert werden. ${ }^{55}$ Der Unterschied der alten Mutageneseverfahren und der natürlich auftretenden Mutationen läge lediglich darin, dass sich die neuen Methoden der genetischen Veränderung besser steuern ließen und zielgerichteter Anwendung fänden. ${ }^{56}$ Durch gerichtete Verfahren könnten demnach Mutationen erzeugt werden, welche sich qualitativ nicht von entsprechend natürlich entstandenen, sog. ungerichteten Verfahren, unterschieden. Es bestehe somit eine rechtliche Ungleichbehandlung wesentlich gleicher Sachverhalte. ${ }^{57}$ Eben deshalb ist das Urteil umstritten, weil diese sog. Punktmutationen nicht nur durch den Menschen

41) Tagesschau: Neue Gentechnik nur unter strengen Auflagen, Stand 28.10.2021, abrufbar unter https://www.tagesschau.de/ ausland/eugh-gentechnik-recht-101.html.

42) Faltus, ZUR 2018, 525.

43) Andersen/Schreiber, in: Vöneky, Freiburger Informationspapiere zum Völkerrecht und Öffentlichen Recht, Ausgabe 11/2019: „Genome Editing“ vor dem EuGH und seine Folgen, S. 7.

44) Andersen/Schreiber, in: Vöneky, Freiburger Informationspapiere zum Völkerrecht und Öffentlichen Recht, Ausgabe 11/2019: „Genome Editing“" vor dem EuGH und seine Folgen, S. 7.

45) EuGH, Urt. v. 25. 7.2018 - C-528/16, ECLI:EU:C:2018:583.

46) Buchholz/Willand, LMuR 2019, 45.

47) Bayerisches Staatsministerium für Umwelt und Verbraucherschutz (Hrsg.): Die rechtliche Situation bei der Nutzung von Genscheren, Stand 28.10.2021, abrufbar unter https://www.stmuv.bayern.de/ themen/biotechnologie/nmt/rechtliche_situation.htm.

48) Bayerisches Staatsministerium für Umwelt und Verbraucherschutz (Hrsg.): Die rechtliche Situation bei der Nutzung von Genscheren, Stand 28.10.2021, abrufbar unter https://www.stmuv.bayern.de/ themen/biotechnologie/nmt/rechtliche_situation.htm.

49) EuGH, Urt. v. 25. 7.2018 - C-528/16, ECLI:EU:C:2018:583.

50) Faktisch findet die Gen-Schere aber eher wenig Anwendung: Nach dem Joint Research Center der EU bestehen weltweit zwar mehr als 400 Forschungsprojekte, allerdings gibt es nur eine einzige Pflanze im Anbau, deren Erbgut per Gen-Schere verändert wurde und zwar eine Sojabohne mit verändertem Ölsäuremuster., siehe: Tagesschau: Gen-Schere spaltet Landwirtschaft, Stand 28.10.2021, abrufbar unter https://www.tagesschau.de/wirtschaft/technologie/gentechnik-genom-editing-101.html.

51) Bayerisches Staatsministerium für Umwelt und Verbraucherschutz (Hrsg.): Die rechtliche Situation bei der Nutzung von Genscheren, Stand 28.10.2021, abrufbar unter https://www. stmuv.bayern.de/themen/biotechnologie/nmt/rechtliche_situation.htm.

52) Faltus, Stellungnahme im Rahmen der Sachverständigenanhörung durch den Ausschuss für Ernährung und Landwirtschaft im Deutschen Bundestag, Stand 28.10.2021, abrufbar unter https:// www.bundestag.de/resource/blob/666020/3ce7f0cd0ebdc3dd 6dc19b3473a77790/Stellungnahme_A_Faltus-data.pdf, S. 4.

53) Faltus, ZUR 2018, 526.

54) Faltus, ZUR 2018, 526.

55) Faltus, Stellungnahme im Rahmen der Sachverständigenanhörung durch den Ausschuss für Ernährung und Landwirtschaft im Deutschen Bundestag, Stand 28.10.2021, abrufbar unter https:// www.bundestag.de/resource/blob/666020/3ce7f0cd0ebdc3dd 6dc19b3473a77790/Stellungnahme_A_Faltus-data.pdf, S. 4.

56) Faltus, Stellungnahme im Rahmen der Sachverständigenanhörung durch den Ausschuss für Ernährung und Landwirtschaft im Deutschen Bundestag, Stand 28.10.2021, abrufbar unter https:// www.bundestag.de/resource/blob/666020/3ce7f0cd0ebdc3dd 6dc19b3473a77790/Stellungnahme_A_Faltus-data.pdf, S. 4

57) Faltus, Stellungnahme im Rahmen der Sachverständigenanhörung durch den Ausschuss für Ernährung und Landwirtschaft im Deutschen Bundestag, Stand 28.10.2021, abrufbar unter https:// www.bundestag.de/resource/blob/666020/3ce7f0cd0ebdc3dd 6dc19b3473a77790/Stellungnahme_A_Faltus-data.pdf, S. 4. 
herbeigeführt werden könnten, sondern zudem auch natürlicherweise in der DNA auftreten. Mutationen entstünden unzählige Male, beispielsweise durch UV-Strahlung auf dem Acker. Auch werde seit den 1920er Jahren durch mutationsauslösende Agenzien, wie beispielsweise Strahlung oder chemische Stoffe, versucht, die bereits vorhandene natürliche Mutationsrate zu erhöhen. ${ }^{58}$ So würden ungerichtete Mutagenese-Verfahren in der Regel dazu führen, dass das gesamte Genom eines Organismus betroffen ist, was eine Vielzahl an zufälligen Mutationen zur Folge haben kann. ${ }^{59}$ Momentan würden diese Pflanzen durch natürliche Punktmutationen ohne Untersuchung regulär in den Nahrungskreislauf des Menschen gelangen. ${ }^{60}$ Das stößt aus naturwissenschaftlicher Sicht auf Unverständnis: Sollte das Ergebnis solcher natürlich auftretender Punktmutationen eine Gefährdung für die Menschen oder die Umwelt darstellen, müssten diese konsequenterweise auch streng kontrolliert und reguliert werden. Dies sei bis heute aber nicht der Fall. Natürlich auftretende Punktmutationen gelten als sicher. Zudem würde eine solche engmaschige Überwachung hin auf zufällige Mutationen einen zu hohen wirtschaftlichen Aufwand darstellen. ${ }^{61}$ Die naturwissenschaftliche Kritik an dem Urteil ist somit nicht zu verkennen: Die Vereinheitlichung von verschiedenen Mutageneseverfahren scheint nicht nachvollziehbar.

\subsubsection{Rechtswissenschaftliche Kritik}

Andere, vor allem rechtswissenschaftliche Stimmen, sagen, die ungerichteten Mutageneseverfahren sollten nicht reguliert werden, weil es gerade der menschliche Beitrag zu einer Handlung sei, der über die (Nicht-)Anwendbarkeit von Recht entscheide. ${ }^{62}$ Dies lasse das Gleichsetzen von gerichteten und ungerichteten Verfahren außer Acht. ${ }^{63}$ Eine rechtliche Bewertung ergebe sich aber nur, wenn ein Mensch an einem Ereignis ursächlich mitgewirkt habe. Bei ungerichteten Mutageneseverfahren ist dies aber eben nicht der Fall, so dass das Argument, bei gerichteten Mutationen auf den ,naturidentischen Charakter" abstellen zu können, nicht überzeugen könne. ${ }^{64}$ Dieses Argument kann aber in einer solchen Absolutheit keinen Bestand haben. Nur der menschliche Eingriff allein kann nicht entscheiden, ob Recht zur Anwendung kommt oder kommen sollte oder nicht. Um Gefahren einzuschränken ist es im Rechtssystem der modus operandi Schutzvorschriften gelten zu lassen, welche regulativ einwirken, auch wenn die Gefahrenquelle an sich nicht von Menschen geschaffen wurde. Es erscheint nicht abwegig zu versuchen beispielsweise mutierte Nahrungsmittel zu selektieren, unabhängig davon, ob Ursache die UV-Strahlen der Sonne oder menschliche Punktmutation war. Rechtswissenschaftlich sei die Entscheidung des EuGH zudem methodisch anzweifelbar. Es werde aus dem Wortlaut des europäischen Gesetzgebers nicht ersichtlich, warum anscheinend nur Mutageneseverfahren von der Richtlinie ausgenommen - und somit privilegiert - sind, welche bis 2001 Anwendung fanden. Hätte der europäische Gesetzgeber nicht ausnahmslos alle Mutageneseverfahren privilegieren wollen, hätte er eine Aufzählung bestimmter Verfahren vornehmen können. Das hat er aber nicht getan, obwohl bereits 2001 verschiedene Mutageneseverfahren etabliert waren und somit eine Differenzierung hätte vorgenommen werden können. ${ }^{65}$ Es erschiene naheliegender, dass der Begriff der Mutagenese im Gegensatz zur Transgenese vom Gesetzgeber verstanden werden sollte. ${ }^{66}$

\subsubsection{Bewertung}

Nichtsdestotrotz kann in Bezug auf GVO ein Risiko nicht vollends ausgeschlossen werden, da aufgrund der Neuartigkeit der Züchtungstechnik noch keine in Langzeitbeobachtungen belegten Erkenntnisse vorliegen. ${ }^{67}$ Zwar sind in Hinblick auf die klassischen GVO die Wirkungspfade bekannt, allerdings stellen die Risikobewertungen ein hohes Unsicherheitselement dar. ${ }^{68}$

Zwar sind die neuen Mutageneseverfahren präziser. Allerdings können auch hier Fehler auftreten: Bei dem neuen
Mutageneseverfahren CRISPR-Cas beispielsweise, besteht das Risiko, dass es beim Schnitt innerhalb der Zelle zu Fehlern kommen kann, welche das Genom verändern oder ausschalten kann. ${ }^{69}$ Zudem muss der rasante Fortschritt in der Entwicklung immer neuer Mutageneseverfahren beachtet werden, gerade im Vergleich zur herkömmlichen Zufallsmutagenese. Vor diesem Hintergrund wäre es aufgrund der Unübersichtlichkeit und Unkontrollierbarkeit unverantwortlich, sollten neue Verfahren unter die Ausnahmerichtlinie fallen. Trotz dieser eben beschriebenen bestehender Ungereimtheiten ist somit vor dem Hintergrund des Vorsorgeprinzips der Auslegung des EuGH bei seiner MutageneseEntscheidung zuzustimmen, macht aber dennoch deutlich, dass die naturwissenschaftlichen Komponenten zu wenig in der europäischen Rechtsprechung Beachtung finden. Das gilt vor allem vor der Tatsache, dass der EuGH sich darauf gestützt hat, dass Verfahren nur ausgenommen werden können, die ,seit langem als sicher gelten“ (17. Erwägungsgrund der EU-Freisetzungsrichtlinie 2001/17/EG). Nach dieser Argumentation könnte aufgrund der zeitlichen Diskrepanz kein einziges neues Verfahren zugelassen werden. ${ }^{70}$

\subsection{Sog. „Gene Drives“ als Beispiel fortschreitender synthetischer Biologie}

Ein weiterer gentechnischer Bereich, welcher sich technisch rasant fortentwickelt, ist der der synthetischen Biologie.

\subsubsection{Synthetische Biologie}

Der neue Forschungsbereich der synthetischen Biologie als Molekularbiologie teilt sich sowohl Methoden, als auch

58) Andersen/Schreiber, in: Vöneky, Freiburger Informationspapiere zum Völkerrecht und Öffentlichen Recht, Ausgabe 11/2019: ,Genome Editing" vor dem EuGH und seine Folgen, S. 6.

59) Andersen/Schreiber, in: Vöneky, Freiburger Informationspapiere zum Völkerrecht und Öffentlichen Recht, Ausgabe 11/2019: „Genome Editing" vor dem EuGH und seine Folgen, S. 6.

60) Faltus, Stellungnahme im Rahmen der Sachverständigenanhörung durch den Ausschuss für Ernährung und Landwirtschaft im Deutschen Bundestag, Stand 28.10.2021, abrufbar unter https:// www.bundestag.de/resource/blob/666020/3ce7f0cd0ebdc3dd 6dc19b3473a77790/Stellungnahme_A_Faltus-data.pdf, S. 4.

61) Faltus, ZUR 2018, 528.

62) Spranger, Stellungnahme im Rahmen der Sachverständigenanhörung durch den Ausschuss für Ernährung und Landwirtschaft im Deutschen Bundestag, Stand 28.10.2021, abrufbar unter https://www.jura.uni-bonn.de/fileadmin/Fachbereich_Rechtswissenschaft/Einrichtungen/Lehrstuehle/Herdegen/Spranger/ Stellungnahme_Spranger.pdf, S. 3.

63) Spranger, Stellungnahme im Rahmen der Sachverständigenanhörung durch den Ausschuss für Ernährung und Landwirtschaft im Deutschen Bundestag, Stand 28.10.2021, abrufbar unter https://www.jura.uni-bonn.de/fileadmin/Fachbereich_Rechtswissenschaft/Einrichtungen/Lehrstuehle/Herdegen/Spranger/ Stellungnahme_Spranger.pdf, S. 3.

64) Spranger, Stellungnahme im Rahmen der Sachverständigenanhörung durch den Ausschuss für Ernährung und Landwirtschaft im Deutschen Bundestag, Stand 28.10.2021, abrufbar unter https://www.jura.uni-bonn.de/fileadmin/Fachbereich_Rechtswissenschaft/Einrichtungen/Lehrstuehle/Herdegen/Spranger/ Stellungnahme_Spranger.pdf, S. 3 .

65) Andersen/Schreiber, in: Vöneky, Freiburger Informationspapiere zum Völkerrecht und Öffentlichen Recht, Ausgabe 11/2019: ,Genome Editing" vor dem EuGH und seine Folgen, S. 14.

66) Andersen/Schreiber, in: Vöneky, Freiburger Informationspapiere zum Völkerrecht und Öffentlichen Recht, Ausgabe 11/2019: ,,Genome Editing" vor dem EuGH und seine Folgen, S. 14.

67) Mechel, in: Koch/Hofmann/Reese, Handbuch Umweltrecht, 5. Aufl. 2018, \11, Rdnr. 20.

68) Mechel, in: Koch/Hofmann/Reese, Handbuch Umweltrecht, 5. Aufl. 2018, §11, Rdnr. 20.

69) Seitz, EuZW 2018, 759

70) Beck, EurUP 2019, 246. 
Verfahrensweisen mit der Gentechnik. ${ }^{71}$ Die synthetische Biologie zielt darauf $\mathrm{ab}$, neue organische Komponenten und Organismen $\mathrm{zu}$ erzeugen und $\mathrm{zu}$ beherrschen. ${ }^{72}$ Die synthetische Biologie ist dabei eine relativ junge Technologie. ${ }^{73}$ Die gezielte Konstruktion von Zellen bestimmter Eigenschaften geschieht im Gegensatz zur Gentechnik, die einzelne Gene in bereits existierende Organismen einzubringen versucht. ${ }^{74}$ Mit der synthetischen Biologie erweitert sich somit der Anwendungsbereich der Gentechnik.

\subsubsection{Gene Drives}

Besonders im Fokus stehen die sog. Gene Drives. „Großen Teilen der allgemeinen Öffentlichkeit sind die Risiken, aber auch Chancen dieses Vererbungsturbos derzeit noch völlig unbekannt", äußerte sich 2018 der Vorsitzende des Deutschen Ethikrates, Peter Dabrock. ${ }^{75}$ Unter Gene Drives versteht man genetische Elemente, welche die Vererbungsrate eines bestimmten Merkmals erhöhen, womit eine bestimmte Eigenschaft in freilebenden Populationen verändert oder reduziert wird. ${ }^{76}$ Innerhalb von 15-20 Generationen könnte somit ein erwünschtes Merkmal in einer ganzen Population verbreitet werden, wie beispielsweise die Malariaübertragung von Mücken gestoppt werden. ${ }^{77}$ Das Potential der Gene Drives liegt somit in der Bekämpfung übertragbarer Krankheiten sowohl beim Menschen als auch bei Tieren, sowie im Anwendungsbereich im Naturschutz und der Landwirtschaft. ${ }^{78}$

\subsubsection{Einordnung}

Politische Parteien wie Bündnis 90/Die Grünen beispielsweise wollen allerdings die Anwendung von Gene Drives laut ihrem Wahlprogramm zur Bundestagswahl 2021 komplett ausschließen. ${ }^{79}$ Auch das Europäische Parlament bekräftigte in seiner Plenarabstimmung am 8.6.2021 seine vorsorgeorientierte Haltung gegenüber dem Einsatz des

71) Sturma, in: Sturma/Lanzerath, Synthetische Biologie, Naturwissenschaftliche, rechtliche und ethische Aspekte, Bd. 20, 2020, S. 9

72) Sturma, in: Sturma/Lanzerath, Synthetische Biologie, Naturwissenschaftliche, rechtliche und ethische Aspekte, Bd. 20, 2020, S. 9

73) Schweizerische Akademie der Technischen Wissenschaften (SATW): Synthetische Biologie. Eine neue Ingenieurwissenschaft entsteht, Stand 28.10.2021, abrufbar unter https://www. satw.ch/fileadmin/user_upload/documents/02_Themen/06_ Rohstoffe/SATW-Synthetische_Biologie-DE.pdf.

74) Sturma, in: Sturma/Lanzerath, Synthetische Biologie, Naturwissenschaftliche, rechtliche und ethische Aspekte, Bd. 20, 2020, S. 9.

75) Deutsches Ärzteblatt: Gene Drive: Das Ende der Vererbungsregeln, Stand 28.10.2021, abrufbar unter https://www.aerzteblatt. de/archiv/200434/Gene-Drive-Das-Ende-der-Vererbungsregeln.

76) Akademie der Naturwissenschaften Schweiz (SCNAT): Gene Drives: Nutzen, Risiken und mögliche Anwendungen, Swiss Academies Factsheets Vol 15, No. 4, 2020, abrufbar unter: https://naturwissenschaften.ch/synthetic-biology-explained/applications/ gene_drive/uuid/i/045a3073-e301-5215-a0a0-3ca3d5b85a78Gene_Drives\%3A_Nutzen\%2C_Risiken_und_m\%C3\%B6gliche_Anwendungen.

77) Akademie der Naturwissenschaften Schweiz (SCNAT): Gene Drives: Nutzen, Risiken und mögliche Anwendungen, Swiss Academies Factsheets Vol 15, No. 4, 2020, abrufbar unter https://naturwissenschaften.ch/synthetic-biology-explained/applications/ gene_drive/uuid/i/045a3073-e301-5215-a0a0-3ca3d5b85a78Gene_Drives\%3A_Nutzen\%2C_Risiken_und_m\%C3\%B6gliche_Anwendungen.

78) Akademie der Naturwissenschaften Schweiz (SCNAT): Gene Drives: Nutzen, Risiken und mögliche Anwendungen, Swiss Academies Factsheets Vol 15, No. 4, 2020, abrufbar unter https://naturwissenschaften.ch/synthetic-biology-explained/applications/ gene_drive/uuid/i/045a3073-e301-5215-a0a0-3ca3d5b85a78Gene_Drives\%3A_Nutzen\%2C_Risiken_und_m\%C3\%B6gliche_Anwendungen.

79) Bündnis 90/Die Grünen, Bundestagswahlprogramm, Stand 28.10.2021, abrufbar unter https://cms.gruene.de/uploads/documents/Wahlprogramm-DIE-GRUENEN-Bundestagswahl2021_barrierefrei.pdf, S. 43.
Gene Drive. ${ }^{80}$ Grund zur Skepsis bestehe unter anderem in der potentiellen Unumkehrbarkeit der einmal freigesetzten Gene Drives. Diese könnten nur schwer oder gar nicht mehr rückrufbar sein. ${ }^{81}$ Auch die unbeabsichtigte Übertragung auf eine andere eng verwandte Art könnte problematisch sein. ${ }^{82}$ Aus einem Bericht der Zentralen Kommission für die biologische Sicherheit (ZKBS) zur synthetischen Biologie in Deutschland aus dem Jahre 2012 geht aber hervor, dass die aktuellen Forschungsansätze in Deutschland vom Gentechnikgesetz erfasst werden und somit kein zusätzliches biosicherheits-spezifisches Gefährdungspotential bergen, welches über das von „klassischen“ gentechnischen Versuchen hinausgeht. ${ }^{83}$ Mit Ausnahme der Synthese von Nukleinsäure wird demnach die gesamte Forschung vom Gentechnikgesetz erfasst. ${ }^{84}$ Diese Erkenntnis bestätigte ein zweiter weltweiter Bericht aus dem Jahr 2018. ${ }^{85}$ Die ZKBS betont, dass kein Änderungsbedarf an der momentanen Rechtslage bestehe. ${ }^{86}$

Ausgehend von der großen Bedeutung der synthetischen Biologie, die hohes Potenzial aufweist, kann zudem davon ausgegangen werden, dass es in den nächsten Jahren zu einer ,regelrechten Biologisierung der Wirtschaft" kommen könnte. ${ }^{87}$ Es stellen sich Fragen was erforscht und (auch aus ethischer Sicht) synthetisiert werden darf, welche Daten im Zuge des Dual-Use missbraucht werden könnten und damit nicht der Öffentlichkeit zugänglich gemacht werden

80) Zukunftsstiftung Landwirtschaft: Europäisches Parlament fordert Verbot der Freisetzung von Gene Drive Organismen, Stand 28.10.2021, abrufbar unter https://www.stop-genedrives.eu/ europaeisches-parlament-fordert-verbot-der-freisetzung-vongene-drive-organismen/.

81) Akademie der Naturwissenschaften Schweiz (SCNAT), Gene Drives, Nutzen, Risiken und mögliche Anwendungen, Swiss Academies Factsheets Vol 15, No. 4, 2020, Stand 28.10.2021, abrufbar unter: https://naturwissenschaften.ch/synthetic-biology-explained/applications/gene_drive/uuid/i/045a3073e301-5215-a0a0-3ca3d5b85a78-Gene_Drives\%3A_Nutzen\% 2C_Risiken_und_m\%C3\%B6gliche_Anwendungen.

82) Akademie der Naturwissenschaften Schweiz (SCNAT), Gene Drives, Nutzen, Risiken und mögliche Anwendungen, Swiss Academies Factsheets Vol 15, No. 4, 2020, Stand 28.10.2021, abrufbar unter https://naturwissenschaften.ch/synthetic-biology-explained/applications/gene_drive/uuid/i/045a3073e301-5215-a0a0-3ca3d5b85a78-Gene_Drives\%3A_Nutzen\% 2C_Risiken_und_m\%C3\%B6gliche_Anwendungen.

83) Bundesamt für Verbraucherschutz und Lebensmittelsicherheit: Monitoring der Synthetischen Biologie in Deutschland, 1. Zwischenbericht der Zentralen Kommission für Biologische Sicherheit, Stand 28.10.2021, abrufbar unter https://www.zkbsonline.de/ZKBS/SharedDocs/Downloads/01_Allgemeine\% 20Stellungnahmen/01_Allgemeine\%20Themen/1._Bericht_ ZKBS_Synthetische_Biologie_in_Deutschland_2012.pdf?_blob $=$ publicationFile $\& \mathrm{v}=9, \mathrm{~S} .11$.

84) Bundesamt für Verbraucherschutz und Lebensmittelsicherheit, Monitoring der Synthetischen Biologie in Deutschland, 1. Zwischenbericht der Zentralen Kommission für Biologische Sicherheit, Stand 28.10.2021, abrufbar unter https://www.zkbsonline.de/ZKBS/SharedDocs/Downloads/01_Allgemeine\% 20Stellungnahmen/01_Allgemeine\%20Themen/1._Bericht_ ZKBS_Synthetische_Biologie_in_Deutschland_2012.pdf? blob $=$ publicationFile $\& \mathrm{v}=9, \mathrm{~S} .11$.

85) Zentrale Kommission für die Biologische Sicherheit, GeneDrive-Systeme, Stand 28.10.2021, abrufbar unter https://www. zkbs-online.de/ZKBS/DE/Meta/Fokusthemen/Gene-DriveSysteme/Gene-Drive-Systeme_node.html.

86) Zentrale Kommission für die Biologische Sicherheit, GeneDrive-Systeme, Stand 28.10.2021, abrufbar unter https://www. zkbs-online.de/ZKBS/DE/Meta/Fokusthemen/Gene-DriveSysteme/Gene-Drive-Systeme_node.html.

87) Schweizerische Akademie der Technischen Wissenschaften (SATW), Synthetische Biologie. Eine neue Ingenieurwissenschaft entsteht, Stand 28.10.2021, abrufbar unter https://www. satw.ch/fileadmin/user_upload/documents/02_Themen/06_ Rohstoffe/SATW-Synthetische_Biologie-DE.pdf. 
dürfen. ${ }^{88}$ Welche Lebensformen durch unkontrollierbare Risiken gefährdet sind und wer die Forschungsgelder für welche Art der Forschung aufbringen darf. ${ }^{89}$ Daher gibt es Stimmen in der Literatur, die neue gesetzliche Regelungen aufgrund dieser neuen Technologie fordern. ${ }^{90}$ Das drängendste Problem ist aber wohl die Feldforschung. Gene Drives können nur im Labor sicher getestet werden. Für eine ausreichende Forschung, die Sicherheit gewährleistet, müssten die Gene Drives aber in der Natur getestet werden. Einmal in den Kreislauf gebracht, sind sie nicht mehr rückholbar. Auch die Frage, inwiefern Bürgerinnen und Bürger einem solchen Feldversuch zustimmen müssten oder könnten und wer für eventuelle Haftungsfragen auch langfristig einsteht, sind bisher noch ungeklärt.

\subsection{Lebensmittel aus geklonten Tieren: sog. „Novel Foods“}

Lebensmittel von geklonten Tieren, wie beispielsweise Lachs oder Milch, fallen unter so genannte neuartige Lebensmittel nach der Verordnung (EG) Nr. 258/97, „Novel Food Verordnung". ${ }^{91}$ In der Europäischen Union sind sie damit grundsätzlich verboten und dürfen lediglich in den Verkehr gebracht werden, wenn sie nach behördlicher Prüfung einschließlich Sicherheitsbewertung zugelassen worden sind. ${ }^{92}$ Bislang wurde für Lebensmittel von geklonten Tieren kein Antrag gestellt und damit auch noch keine Zulassung erteilt. ${ }^{93}$ Mangels eines gestellten Antrags dürfen die von Klontieren gewonnenen Lebensmittel somit faktisch in der EU momentan nicht verkauft werden. ${ }^{94}$ Praktisch spielt das Klonen von Tieren in Europa aber bisher auch keine relevante Rolle. ${ }^{95}$ Dies liegt unter anderem daran, dass die Methoden der klassischen Gentechnik gerade bei Nutztieren häufig fehleranfällig, wenig effizient und mit hohen Kosten verbunden sind. ${ }^{96}$ Aktuell wird auf europäischer Ebene diskutiert, ob das Klonen von Nutztieren allgemein gesetzlich verboten werden und ob es ein Einfuhrverbot geklonter Tiere und ih-

88) Schweizerische Akademie der Technischen Wissenschaften (SATW), Synthetische Biologie. Eine neue Ingenieurwissenschaft entsteht, Stand 28.10.2021, abrufbar unter https://www. satw.ch/fileadmin/user_upload/documents/02_Themen/06_ Rohstoffe/SATW-Synthetische_Biologie-DE.pdf.

89) Schweizerische Akademie der Technischen Wissenschaften (SATW), Synthetische Biologie. Eine neue Ingenieurwissenschaft entsteht, Stand 28.10.2021, abrufbar unter https://www. satw.ch/fileadmin/user_upload/documents/02_Themen/06_ Rohstoffe/SATW-Synthetische_Biologie-DE.pdf.

90) Seitz, EuZW 2018, 763.

91) Bundesministerium für Ernährung und Landwirtschaft: Lebensmittel von geklonten Tieren, Stand 28.10.2021, abrufbar unter https://www.bmel.de/DE/themen/verbraucherschutz/lebensmittelsicherheit/spezielle-lebensmittel/fleisch-klontiere.html; jsessionid=33963C44130334DD5DE947CDA50C269A.live842.

92) Bundesministerium für Ernährung und Landwirtschaft, Lebensmittel von geklonten Tieren, Stand 28.10.2021, abrufbar unter https://www.bmel.de/DE/themen/verbraucherschutz/lebensmittelsicherheit/spezielle-lebensmittel/fleisch-klontiere.html; jsessionid=33963C44130334DD5DE947CDA50C269A.live842.

93) Bundesministerium für Ernährung und Landwirtschaft, Lebensmittel von geklonten Tieren, Stand 28.10.2021, abrufbar unter https://www.bmel.de/DE/themen/verbraucherschutz/lebensmittelsicherheit/spezielle-lebensmittel/fleisch-klontiere.html; jsessionid=33963C44130334DD5DE947CDA50C269A.live842.

94) Deutsches Referenzzentrum für Ethik in den Biowissenschaften Geklonte Nutztiere, Stand 28.10.2021, abrufbar unter https:// www.drze.de/im-blickpunkt/gmf/module/geklonte-nutztiere.

95) Tagesspiegel: „Es war richtig, das Klonen zu verbieten“, Stand 28.10.2021, abrufbar unter https://www.tagesspiegel.de/wissen/20-jahre-aechtung-durch-die-eu-es-war-richtig-das-klonen-zu-verbieten/20547770.html.

96) Forum Bio- und Gentechnologie: Gentechnisch veränderte Tiere: Gibt es die schon?, Stand 28.10.2021, abrufbar unter https://www.transgen.de/tiere/996.gentechnisch-veraendertetiere-fragen-antworten.html. rer Nachkommen und Produkte geben sollte. ${ }^{97}$ Am 3. 9. 2020 hat das Europäische Parlament die Kommission aufgefordert, das Klonen von Tieren für die Lebensmittelversorgung zu verbieten. ${ }^{98}$ Trotz der geringen Anwendungszahlen in der Praxis, bestehe eine Notwendigkeit für ein solches Verbot aufgrund von tierschutzrechtlichen Aspekten, Gefährdung der genetischen Vielfalt, dem Verbrauchervertrauen und dem „Image und Charakter des europäischen Landwirtschaftsmodells“. 99 Während der Motivationsgrund der Imageverbesserung fragwürdig erscheint, ist der tierschutzrechtliche und ethische Aspekt nicht zu unterschätzen, vor allem vor dem Hintergrund der Staatszielbestimmung des Tierschutzes aus Art. 20a GG. Geklonte Rinder und Schweine scheinen von normaler gesundheitlicher Kondition zu sein; allerdings kann es auch zu schweren Gesundheitsschäden und abnormalen Entwicklungen kommen, wenn während der sog. Reprogrammierungsphase beim Klonungsvorgang Fehler auftreten. ${ }^{100}$ Häufig treten auch Atemprobleme, Herz-Kreislauf-Versagen, Immundefekte und Leberversagen auf. ${ }^{101}$

Fraglich ist dennoch, ob durch ein solches Verbot die Entwicklung sinnvoller Anwendungen blockiert werden könnte. In den USA beispielsweise ist es durch die Verbindung von Klonen und der Genome Editing-Technik CRISPR gelungen, hornlose Rinder zu generieren. ${ }^{102} \mathrm{Um}$ die Strapazen der Stallhaltung zu minimieren war und ist es nämlich gängige Praxis die Rinder mechanisch zu enthornen. ${ }^{103}$ Der Ansatz, die Rinder bereits ohne Hörner zu züchten, ist im Gegensatz zur mechanischen Enthornung zwar ein völlig schmerzfreier, ${ }^{104}$ kann aber - wie oben bereits dargestellt - erhebliche Auswirkungen auf das Tierwohl durch Komplikationen im Klonprozess haben. Aus tierschutzethischer Perspektive ist das Klonen mithin bedenkenswert und ein Verbot trotz ausbleibendem Klonvorkommen in Deutschland zu begrüßen.

97) CORDIS, Forschungsergebnisse der EU: Europäisches Parlament fordert Kommission auf, das Klonen von Tieren für die Lebensmittelversorgung zu verbieten, Stand 28.10.2021, abrufbar unter https://cordis.europa.eu/article/id/29825-european-parliamenturges-commission-to-ban-animal-cloning-for-food-supply/de.

98) CORDIS, Forschungsergebnisse der EU: Europäisches Parlament fordert Kommission auf, das Klonen von Tieren für die Lebensmittelversorgung zu verbieten, Stand 28.10.2021, abrufbar unter https://cordis.europa.eu/article/id/29825-european-parliamenturges-commission-to-ban-animal-cloning-for-food-supply/de.

99) CORDIS, Forschungsergebnisse der EU: Europäisches Parlament fordert Kommission auf, das Klonen von Tieren für die Lebensmittelversorgung zu verbieten, Stand 28.10.2021, abrufbar unter https://cordis.europa.eu/article/id/29825-european-parliament-urges-commission-to-ban-animal-cloningfor-food-supply/de.

100) CORDIS, Forschungsergebnisse der EU: Europäisches Parlament fordert Kommission auf, das Klonen von Tieren für die Lebensmittelversorgung zu verbieten, Stand 28.10.2021, abrufbar unter https://cordis.europa.eu/article/id/29825-european-parliament-urges-commission-to-ban-animal-cloningfor-food-supply/de.

101) Redaktionsnetzwerk Deutschland: 25 Jahre nach Dolly: Wo wird die Klontechnologie heute eingesetzt?, Stand 28.10.2021, abrufbar unter https://www.rnd.de/wissen/klon-schaf-dolly25-jahre-danach-wo-wird-die-klontechnologie-heute-eingesetzt-G7WD7YGCVFFUVANSOOOZDMBFUI.html.

102) Tagesspiegel: „Es war richtig, das Klonen zu verbieten“, Stand 28.10.2021, abrufbar unter https://www.tagesspiegel.de/wissen/20-jahre-aechtung-durch-die-eu-es-war-richtig-das-klonen-zu-verbieten/20547770.html.

103) Tagesspiegel: „Es war richtig, das Klonen zu verbieten“, Stand 28.10.2021, abrufbar unter https://www.tagesspiegel.de/wissen/20-jahre-aechtung-durch-die-eu-es-war-richtig-das-klonen-zu-verbieten/20547770.html.

104) Tagesspiegel: „Es war richtig, das Klonen zu verbieten“, Stand 28.10.2021, abrufbar unter https://www.tagesspiegel.de/wissen/20-jahre-aechtung-durch-die-eu-es-war-richtig-das-klonen-zu-verbieten/20547770.html. 


\subsection{Aufkommen von „Do-It-Yourself“-Biologie}

Obwohl Biologiebaukästen (meist für Kinder und Jugendliche) keine neue Erscheinung sind, stellen die im Internet erhältlichen „Do-It-Yourself“-Biologiebaukästen eine Neuerung dar. Mit diesen DIY-Kästen ist es Privatpersonen möglich, das Erbgut von Organismen zu verändern, beispielsweise von E. coli Bakterien. ${ }^{105}$ Sollten - je nach Art des Baukastens - aber GVO enthalten sein oder GVO erzeugt werden, fällt das Vorhaben unter das Gentechnikgesetz gemäß $\$ 8$ Abs. 1 S. 1 GenTG und ist damit reglementiert. Solche Arbeiten dürfen laut aktueller Rechtslage nur in behördlich überwachten Laboren unter Aufsicht vorgenommen werden. Bei Verstoß drohen nach $\$ 38$ Abs. 1 Nr. 2 i.V.m. Abs. 2 GenTG eine Geldbuße bis zu fünfzigtausend Euro. Sollten bei diesen Experimenten GVO freigesetzt werden, droht gemäß $\$ 39$ Abs. 2 Nr. 1 GenTG sogar eine Freiheitsstrafe bis zu drei Jahren oder eine Geldstrafe. Die Gefahren von solchen privaten Gentechnikexperimenten sind nicht zu unterschätzen. Sog. Biohacker können Krankheitserreger wie Viren oder Bakterien modifizieren. ${ }^{106}$ Die strengen Regularien des Gentechnikrechts - anders als beispielsweise in den USA - scheinen somit ein adäquates Mittel zu sein, um Risiken zu minimieren. Beim Kit des amerikanischen Herstellers „Odin“ beispielsweise ist Ziel des Experiments für Amateure, antibiotikaresistente Keime zu erzeugen. ${ }^{107}$ Vor der Tatsache, dass antibiotikaresistente Keime weltweit zunehmen, scheint es angebracht zu verhindern, dass solche GVO in die Umwelt gelangen.

\section{Perspektiven}

Weitere hochumstrittene und in Zukunft noch bedeutsamere Verfahren sind neben der Bioinformatik auch die Laborrobotik. Diese sind technische Verfahren, die sich schnell weiterentwickeln. Vor allem vor dem Hintergrund kontinuierlich wachsender Möglichkeiten der Gentechnik ist eine differenzierte und präzise (europäische) Gesetzgebung gefragter denn je. Auch aus verfassungsrechtlicher Perspektive des Art. 20a GG gilt es, in Verantwortung zukünftiger Generationen zu handeln und die natürlichen Lebensgrundlagen zu schützen. ${ }^{108}$ Gerade im Bereich der Gentechnik, welche in elementare Strukturen des Lebens eingreift und die Folgen unter Umständen schwer oder gar nicht wieder rückgängig zu machen sind. ${ }^{109}$ Auch der grundsätzliche Konflikt zwischen der vorsorgeorientierten Haltung auf der einen und Innovationen auf der anderen Seite, scheint noch ungelöst. Alle dargestellten aktuellen Probleme weisen darauf hin, dass die europäische und deutsche Justiz in naturwissenschaftlichen Fragen oftmals an funktionelle und fachliche Grenzen stößt. ${ }^{110}$ Ein Ansatzpunkt dieses Problem zu lösen, könnte in einer unabhängigen Risikoforschung gentechnischer Vorhaben gesehen werden, welche bislang faktisch kaum existiert. ${ }^{111} \mathrm{Da}$ sich Saatgutunternehmen oftmals weigern, gentechnisch verändertes Saatgut zur Forschung zur Verfügung zu stellen, könnte ein zwar radikaler, aber wohl effektiver Ansatzpunkt sein, nur noch Produkte auf den EU Markt kommen $\mathrm{zu}$ lassen, deren Hersteller bei einer unabhängigen Risikoforschung kooperieren. ${ }^{112}$ Dann könnten die Gerichte und die Behörden aufgrund einer besseren und transparenteren Datenlage entscheiden. Die Debatte um Gentechnik ist nicht neu, seit 30 Jahren wird über Sinn und Gefahren von Gentechnik diskutiert. ${ }^{113}$ Die rasanten technischen Fortschritte der letzten Jahre lassen eine umfassende gesellschaftliche Diskussion und angepasste rechtliche Regelung aber noch einmal drängender erscheinen.

Bei der Gentechnik hat die Entfaltung ihrer Möglichkeiten bei gleichzeitiger Minimierung ihrer Risiken einen allgemeinen doppelten Zukunftsbezug: Es geht maßgeblich um künftige Freiheitsentfaltung für die derzeitige und künftige Generation. Diese Freiheitsentfaltung bezieht sich einerseits auf die Entwicklung und Produktion gentechnischer Produkte sowie auf künftige Forschungstätigkeiten und andererseits auf den Schutz vor künftigen Gefahren durch gentechnische Produkte und ihrer Entwicklung für die Allgemeinheit und für Dritte. Hier zeigt sich, dass Chancen und Risiken einer neuen Technik letztlich zwei Seiten einer Medaille sind. Ohne Risiko wird es keine Freiheit der Technikentfaltung geben, also eine Wahrnehmung der Chancen neuer Techniken nicht möglich sein.

Open Access. Dieser Artikel wird unter der Creative Commons Namensnennung 4.0 International Lizenz veröffentlicht, welche die Nutzung, Vervielfältigung, Bearbeitung, Verbreitung und Wiedergabe in jeglichem Medium und Format erlaubt, sofern Sie den/die ursprünglichen Autor(en) und die Quelle ordnungsgemäß nennen, einen Link zur Creative Commons Lizenz beifügen und angeben, ob Änderungen vorgenommen wurden.

Die in diesem Artikel enthaltenen Bilder und sonstiges Drittmaterial unterliegen ebenfalls der genannten Creative Commons Lizenz, sofern sich aus der Abbildungslegende nichts anderes ergibt. Sofern das betreffende Material nicht unter der genannten Creative Commons Lizenz steht und die betreffende Handlung nicht nach gesetzlichen Vorschriften erlaubt ist, ist für die oben aufgeführten Weiterverwendungen des Materials die Einwilligung des jeweiligen Rechteinhabers einzuholen.

Weitere Details zur Lizenz entnehmen Sie bitte der Lizenzinformation auf http://creativecommons.org/licenses/by/4.0/deed.de.

Open Access funding enabled and organized by Projekt DEAL.

105) Bundesamt für Verbraucherschutz und Lebensmittelsicherheit Gentechnik mit Biologiebaukästen: Einfach, aber möglicherweise strafbar, Stand 28.10.2021, abrufbar unter https://www. bvl.bund.de/SharedDocs/Fachmeldungen/06_gentechnik/ 2017/2017_01_25_DIY-Kits.html.

106) In Datenbanken wie GenBank kann jeder die Sequenzen von Genen abrufen, auch die gefährlicher Erreger wie Ebola oder der Spanischen Grippe; Neue Züricher Zeitung: Gentechnik gehört nicht ins Kinderzimmer, Stand 28.10.2021, abrufbar unter https://www.nzz.ch/wissenschaft/genetik-baukastengentechnik-gehoert-nicht-ins-kinderzimmer-ld.1296534.

107) Zentrale Kommission für Biologische Sicherheit: Stellungnahme der ZKBS zur Risikobewertung von im Handel frei erhältlichen Do-it-yourself (DIY)-Kits für gentechnische Experimente, Stand 28.10.2021, abrufbar unter https://www.bvl. bund.de/ZKBS/SharedDocs/Downloads/01_Allgemeine\% 20Stellungnahmen/01_Allgemeine\%20Themen/Do-it-yourself-Kits_2017.pdf?_blob=publicationFile $\& v=$, siehe auch Neue Züricher Zeitung: Gentechnik gehört nicht ins Kinderzimmer, Stand 28.10.2021, abrufbar unter https://www. nzz.ch/wissenschaft/genetik-baukasten-gentechnik-gehoertnicht-ins-kinderzimmer-ld.1296534.

108) Mechel, in: Koch/Hofmann/Reese, Handbuch Umweltrecht, 5. Aufl. 2018, \$11 Rdnr. 51, 52.

109) Mechel, in: Koch/Hofmann/Reese, Handbuch Umweltrecht, 5. Aufl. 2018, \$11 Rdnr. 51, 52.

110) Andersen/Schreiber, in: Vöneky, Freiburger Informationspapiere zum Völkerrecht und Öffentlichen Recht, Ausgabe 11/2019: „Genome Editing“ vor dem EuGH und seine Folgen, S. 16.

111) Bundesministerium für Umwelt, Naturschutz und nukleare Sicherheit: BMU-Positionspapier zum Thema Gentechnik in der Landwirtschaft: Für Wahlfreiheit und Vorsorgeprinzip, Stand 28.10.2021, abrufbar unter https://www.bmu.de/fileadmin/ Daten_BMU/Download_PDF/Naturschutz/positionspapier_ neue_gentechnik_bf.pdf, S. 4

112) Bundesministerium für Umwelt, Naturschutz und nukleare Sicherheit: BMU-Positionspapier zum Thema Gentechnik in der Landwirtschaft: Für Wahlfreiheit und Vorsorgeprinzip, Stand 28.10.2021, abrufbar unter https://www.bmu.de/fileadmin/ Daten_BMU/Download_PDF/Naturschutz/positionspapier_ neue_gentechnik_bf.pdf, S. 4 .

113) Härlin, Allzeit achter Tag der Schöpfung, Böll.Thema 2/2019, Stand 28.10.2021, abrufbar unter https://www.boell.de/de/ 2019/04/09/allzeit-achter-tag-der-schoepfung?dimension1= bt_gentechnik, S. 6-7. 